PACS 64.60.Ht, 68.35.Rh, 05.40.+j, 11.10.-7

\title{
SPECIAL SURFACE TRANSITION: MASSIVE FIELD THEORY AND CRITICAL EXPONENTS IN THREE DIMENSIONS
}

\author{
M.SHPOT \\ Institute for Condensed Matter Physics \\ of the Ukrainian National Academy of Sciences \\ 1 Svientsitskii str., UA-290011 Lviv-11, Ukraine
}

Received January 28, 1997

\begin{abstract}
The surface critical behaviour is studied directly in fixed spatial dimensions $d=4-\epsilon<4$ without resort to the $\epsilon$ expansion. Generalization of the massive field theory approach appropriate to the description of the standard semi-infinite $n$-vector model with the surface term $\frac{1}{2} c_{0} \int_{\partial V} \phi^{2}$ is presented. This involves an additive shift of the surface enhancement $c_{0}$ similar to the bare mass shift in superrenormalizable field theories. Explicit two-loop calculations in three space dimensions yield numerical estimates of surface critical exponents of the special phase transition. Our results are in good agreement with the most recent Monte Carlo simulations.
\end{abstract}

\section{Introduction}

Since the early seventies, extraordinary progress in understanding of phase transitions and critical phenomena was achieved owing to the application of the power of field theory and renormalization group (RG) in this region of statistical mechanics [1-4]. General, conceptually appealing theoretical framework was provided for the investigations of physics that happens. The implementation of field theoretical methods has led to high-precision numerical analysis of critical exponents [5-9], universal amplitude combinations and the features of preasymptotic behaviour [10-13] in the theory of infinite-volume, bulk phase transitions.

Substantial achievements were also gained more recently in the theory of surface critical behaviour (for reviews see references [14-16]). An important point to mention is that this subject is much more complicated from both conceptual and technical points of view, being at the same time very rich with respect to actual physical phenomena. A series of modern field-theoretical methods developed and tested originally in the studies of bulk critical phenomena, have subsequently been extended to systems with boundaries. This has led to considerable success in the investigation of surface critical phenomena occurring at bulk critical temperatures. The techniques applied include dimensionality expansions about upper and lower critical dimensions as well as position-space renormalizations and $1 / n$ expansions for models with $n$-component order parameters. One should however admit that much less is known in this field, in comparison to bulk transitions, in what concerns the accurate quantitative theoretical predictions. 
In our recent paper [17] we have initiated the investigation of the critical behaviour at surfaces using the massive field theory $R G$ approach in fixed space dimensions $d$ below the upper critical dimension $d^{*}[18]$. The merits of this approach are well known: pushed to sufficiently high orders of perturbation theory and combined with sophisticated series resummation techniques, it has produced values of bulk critical exponents in htree dimensions $[5,6]$ belonging to the most accurate ones obtained so far by other methods [7-9,21,22]. In a similar fashion the estimates of universal critical amplitude relations $[10,11]$ of comparable precision were obtained. This method was also possible to apply (although with less numerical accuracy) in the calculation of the correlation length amplitude ratio of three-dimensional Ising model [23], in the study of several anisotropic and disordered systems [24-26], and and in general, noninteger dimensions $2 \leqslant d<4$ [27].

In the present paper we present the generalization of the massive field theoretical approach in fixed dimensions to the description of the special transition occurring in semi-infinite systems bounded with a plane surface. We believe that such extension is an important fundamental problem. On the other hand, we have been motivated by persisting discrepancies between the $\epsilon$ expansion numerical estimates [15] and more recent Monte Carlo calculations [28-32] of the crossover surface critical exponent $\Phi$ in threedimensional systems. Thus, having established the general framework, we performed explicit two-loop calculations appropriate to the special surface phase transition occurring in three-dimensional semi-infinite systems at the bulk critical point. The details of pertaining Feynman graph calculations will be communicated elsewhere [33]. Here we present the results of extensive Padé analyses and Padé-Borel resummations of our series expansions. We illustrate the good agreement of our estimates of the surface critical exponents with the most recent results of computer simulations.

In section 2 we start with the representation of the effective Hamiltonian of the semi-infinite n-vector model with surface, and provide the necessary background information.

In section 3 we recall the well-known normalization conditions of the massive field theory for the renormalized vertex functions of the infinitevolume theory, and establish the new normalization conditions appropriate for the correlation functions of semi-infinite systems described in section 2 . We introduce the surface-enhancement shift resembling the additive mass shift in superrenormalizable bulk theories. This is required in order to eliminate divergences associated with the short-distance singularities appearing due to the presence of the surface.

In section 4 we discuss the general aspects of the massive field theory of the special transition. We write down the corresponding Callan-Symanzik equations and consider the non-analytic temperature dependences of the renormalization $Z$ factors at the special point. We construct also the expansion about the multicritical point in terms of small surface-enhancement deviations from its special value. The critical exponents of surface correlations and susceptibility, as well as the crossover exponent are identified in the framework of our general approach.

In section 5 we consider the underlying perturbation theory. After displaying some general features of perturbative expansions we provide explicit and detailed calculation for arbitrary spatial dimensions in the one-loop approximation. Owing to the lack of space, we only quote here our two-loop results at $d=3$ without presenting any calculational details.

Our second-order series expansions provide the basis for the extensive numerical computations of a whole series of the surface critical exponents pertaining to the special transition. In section 6 we discuss and tabulate 
a lot of numerical values resulting from our Padé analyses and Padé-Borel resummations. For completeness and convenience, a table containing recent results of Monte Carlo simulations is given. It displays a good agreement with our theoretical data. What is the most important, this holds also well for the crossover critical exponent $\Phi$ whose numerical value was controversial over the years.

\section{The model}

Let $\boldsymbol{\phi}=\left(\phi^{a}(\mathbf{x})\right)$ be an $n$-vector field defined on the half-space $V=\mathbb{R}_{+}^{d} \equiv$ $\left\{\mathbf{x}=(\mathbf{r}, z) \in \mathbb{R}^{d} \mid \mathbf{r} \in \mathbb{R}^{d-1}, z \geqslant 0\right\}$ bounded by the plane $z=0$, which we denote as $\partial V$. The semi-infinite $n$-vector model is defined by the Euclidean action [15]

$$
\mathcal{H}[\boldsymbol{\phi}]=\int_{V}\left(\frac{1}{2}|\nabla \boldsymbol{\phi}|^{2}+\frac{1}{2} m_{0}^{2} \boldsymbol{\phi}^{2}+\frac{1}{4 !} u_{0}|\boldsymbol{\phi}|^{4}\right)+\int_{\partial V} \frac{1}{2} c_{0} \boldsymbol{\phi}^{2} .
$$

Here $m_{0}^{2}, u_{0}$, and $c_{0}$ are the bare mass, the bare coupling constant, and the bare surface enhancement ${ }^{1}$, respectively.

Adding bulk and surface source terms to the action, one introduces the generating functional

$$
\begin{aligned}
\mathcal{Z} & {\left[\mathbf{J}, \mathbf{J}_{1} ; K, K_{1}\right] } \\
& =\int \mathcal{D} \boldsymbol{\phi} \exp \left[-\mathcal{H}+\int_{V}\left(\mathbf{J} \cdot \boldsymbol{\phi}+\frac{1}{2} K \boldsymbol{\phi}^{2}\right)+\int_{\partial V}\left(\mathbf{J}_{1} \cdot \boldsymbol{\phi}+\frac{1}{2} K_{1} \boldsymbol{\phi}^{2}\right)\right]
\end{aligned}
$$

and the correlation functions

$$
\begin{aligned}
& G^{\left(N, M ; I, I_{1}\right)}\left(\mathbf{x}_{1}, \ldots, \mathbf{R}_{I_{1}}\right) \\
&=\left[\prod_{j=1}^{N} \frac{\delta}{\delta J^{a_{j}}\left(\mathbf{x}_{j}\right)}\right]\left[\prod_{k=1}^{M} \frac{\delta}{\delta J_{1}^{b_{k}}\left(\mathbf{r}_{k}\right)}\right] \\
& {\left.\left[\prod_{l=1}^{I} \frac{\delta}{\delta K\left(\mathbf{X}_{l}\right)}\right]\left[\prod_{m=1}^{I_{1}} \frac{\delta}{\delta K_{1}\left(\mathbf{R}_{m}\right)}\right] \ln \mathcal{Z}\right|_{J=J_{1}=K=K_{1}=0} . }
\end{aligned}
$$

For the functions $G^{(N, M ; 0,0)}$ without $\phi^{2}$-insertions on or off the surface we use the notation $G^{(N, M)}$. The tensorial indices $\left\{a_{j}, b_{k}\right\}$ will be suppressed whenever no confusion is possible. The ultraviolet (uv) singularities of the theory should be assumed to be regularized by means of a large-momentum cutoff $\Lambda$.

We shall also need the (bulk) analogs of these functions for the $|\phi|^{4}$ theory in the infinite space, i.e., with $V=\mathbb{R}^{d}$. The easiest way to define these is the usual one where all boundary terms in the action (1) and the generating functional (2) are dropped, and periodic boundary conditions

\footnotetext{
${ }^{1}$ Upon mapping a semi-infinite (lattice) Ising model with ferromagnetic nearestneighbour interactions of strength $K_{1}$ between surface spins and of strength $K$ elsewhere one finds that $c_{0}$ decreases as $\left(K_{1}-K\right) / K$ increases [15]. For simplicity, we will nevertheless use the term surface enhancement for $c_{0}$, rather than reserving it for $\left(-c_{0}\right)$ or $\left(-c_{0}+\right.$ const $)$.
} 
are chosen. We denote the so-defined bulk analog of $G^{(N, 0 ; I, 0)}\left(\left\{\mathbf{x}_{j}\right\} ;\left\{\mathbf{X}_{l}\right\}\right)$ as $G_{b u l k}^{(N ; I)}\left(\left\{\mathbf{x}_{j}\right\} ;\left\{\mathbf{X}_{l}\right\}\right)$ and introduce their Fourier transforms $\tilde{G}_{b u l k}^{(N ; I)}$ through

$$
\begin{aligned}
& G_{b u l k}^{(N ; I)}\left(\left\{\mathbf{x}_{j}\right\} ;\left\{\mathbf{X}_{l}\right\}\right)= \\
& \quad \int_{\mathbf{q}_{1}, \ldots, \mathbf{Q}_{I}} \tilde{G}_{b u l k}^{(N ; I)}\left(\left\{\mathbf{q}_{j}\right\} ;\left\{\mathbf{Q}_{l}\right\}\right) e^{i\left(\sum_{j} \mathbf{q}_{j} \mathbf{x}_{j}+\sum_{l} \mathbf{Q}_{l} \mathbf{X}_{l}\right)}(2 \pi)^{d} \delta\left(\sum_{j} \mathbf{q}_{j}+\sum_{l} \mathbf{Q}_{l}\right)
\end{aligned}
$$

where the integral on the right-hand side indicates integrations over all $d$-dimensional momenta $\mathbf{q}_{1}, \ldots, \mathbf{Q}_{I}: \int_{\mathbf{q}} \equiv \int d^{d}(q / 2 \pi)$. For the associated standard bulk vertex functions and their Fourier transforms we use the notation $\Gamma_{b u l k}^{(N ; I)}$ and $\widetilde{\Gamma}_{b u l k}^{(N ; I)}$.

In the case of our half-space geometry, where translational invariance is restricted to translations parallel to the surface, it is appropriate to Fourier transform only with respect to all $(d-1)$-dimensional parallel coordinates. We denote the $(d-1)$-dimensional parallel momenta associated with the fields $\phi(\mathbf{x} \notin \partial V)$ and $\phi_{s} \equiv \phi(\mathbf{x} \in \partial V)$ by lower case $\mathbf{p}$ 's, and those associated with the insertions $\phi^{2}$ and $\phi_{s}^{2}$ by upper case $\mathbf{P}$ 's. Parallel Fourier transforms are indicated by a hat; for example, the pair correlation function in this $\mathbf{p} z$ representation is written as $\hat{G}^{(2,0)}\left(\mathbf{p} ; z, z^{\prime}\right)$.

Infinitely far away from the surface all properties must attain their bulk values. Hence the bulk functions $\hat{G}_{b u l k .}^{(N ; I)}$ can be obtained from $\hat{G}^{(N, 0 ; I, 0)}$ by letting all $N+I$ perpendicular coordinates $z_{j} \rightarrow \infty$, keeping all relative coordinates $z_{j k} \equiv z_{i}-z_{k}$ fixed:

$$
\lim _{z_{1}, \ldots, z_{N+I} \rightarrow \infty} \hat{G}^{(N, 0 ; I, 0)}\left(\{\mathbf{p}\} ;\left\{z_{j}\right\}\right)=\hat{G}_{b u l k}^{(N ; I)}\left(\{\mathbf{p}\} ;\left\{z_{j k}\right\}\right),
$$

where $\{\mathbf{p}\}$ here stands for the set of all $N+I$ parallel momenta.

To proceed, it is necessary to recall a few well-known properties of the model (1) [15]. Its phase diagram exhibits a disordered phase (SD/BD), a surface-ordered, bulk-disordered phase ( $\mathrm{SO} / \mathrm{BD})$, and a surface-ordered, bulk-ordered phase (SO/BO), provided $d$ exceeds the lower critical dimension $d_{*}(n)=3-\delta_{n 1}-2 \delta_{n 0}$ for the appearance of a SO/BD phase ${ }^{2}$. The boundaries between these phases are the lines of surface, ordinary, and extraordinary transitions. They meet at a multicritical point, $\left(m_{0}^{2}, c_{0}\right)=$ $\left(m_{0 c}^{2}, c_{0}^{s p}\right)$, called special point and corresponding to the special transition. The ordinary and extraordinary transitions correspond respectively to the portions $c_{0}>c_{0}^{s p}$ and $c_{0}<c_{0}^{s p}$ of the line of bulk criticality $m_{0}^{2}=m_{0 c}^{2}$. The line of surface transitions separates the $\mathrm{SD} / \mathrm{BD}$ from the $\mathrm{SO} / \mathrm{BD}$ phase. At bulk criticality, we thus have three distinct surface transitions - the ordinary, special, and extraordinary. Of these only the ordinary and special transition can be reached from the disordered phase and may be treated by means of our present analysis. In the present paper we restrict ourselves only to the latter type of transition.

The consideration of the disordered phase simplifies the analysis considerably. One does not have to deal with a nonvanishing, and spatially

\footnotetext{
${ }^{2}$ In other words, the dimensionality of space $d$ should be so that the $d-1$ dimensional boundary of the system may independently attain the long-range order (we do not consider more complicated situations occurring in XY models). The special transition discussed just below does not exist in two dimensions in Ising systems $(n=1)$ whereas it still remains in the polymer adsorption $(n=0)[34]$.
} 
varying, order-parameter profile $\langle\phi(\mathbf{x})\rangle$, and the free propagator in the $\mathbf{p} z$ representation takes the relatively simple form

$$
\hat{G}\left(\mathbf{p} ; z, z^{\prime}\right)=\frac{1}{2 \kappa_{0}}\left[e^{-\kappa_{0}\left|z-z^{\prime}\right|}-\frac{c_{0}-\kappa_{0}}{c_{0}+\kappa_{0}} e^{-\kappa_{0}\left(z+z^{\prime}\right)}\right]
$$

with

$$
\kappa_{0}=\sqrt{p^{2}+m_{0}^{2}} .
$$

The translationally invariant first term on the right-hand side of (6) is the free bulk propagator.

The perturbation series of the functions (3) in terms of the free propagator (6) can be regularized by setting $\hat{G}\left(\mathbf{p} ; z, z^{\prime}\right)=0$ for $|\mathbf{p}|>\Lambda$. Alternatively, we shall use in the following the dimensional regularization of the theory.

\section{Normalization Conditions of the Massive Field Theory}

Our aim is to study surface critical behaviour at a bulk critical point. Therefore, a necessary property we ought to require from our approach is that the bulk critical behaviour be treated appropriately. A convenient way to achieve this is to choose it in such a manner that it reduces for all bulk quantities to a well-established standard procedure. In our case this will be the conventional one based on normalization conditions (see, e. g., $[35,36,19,20,3])$. Alternatively, one could choose a massive approach based on minimal subtraction of poles, as described for the bulk case by Schloms and Dohm [12].

\subsection{Bulk normalization conditions}

Starting from the bare bulk vertex functions $\Gamma_{b u l k}^{(N, I)}\left(. ; m_{0}^{2},, u_{0}\right)$, we perform a mass shift

$$
m_{0}^{2}=m^{2}+\delta m^{2}(\epsilon)
$$

and introduce (uv-finite) renormalization factors $Z_{\phi}(u), Z_{\phi^{2}}(u), Z_{u}(u)$ as well as a renormalized dimensionless coupling constant $u$ and renormalized fields such that

$$
\phi=\left[Z_{\phi}(u)\right]^{1 / 2} \phi_{r e n}, \quad \phi^{2}=\left[Z_{\phi^{2}}(u)\right]^{-1}\left[\phi^{2}\right]_{r e n}, \quad u_{0}=Z_{u}(u) m^{\epsilon} u .
$$

The mass shift and the renormalization factors are fixed through the standard normalization conditions

$$
\begin{gathered}
\left.\tilde{\Gamma}_{b u l k, r e n}^{(2)}(\mathbf{q} ; u, m)\right|_{\mathbf{q}=\mathbf{0}}=m^{2}, \\
\left.\frac{\partial}{\partial q^{2}} \tilde{\Gamma}_{b u l k, r e n}^{(2)}(\mathbf{q} ; u, m)\right|_{\mathbf{q}=\mathbf{0}}=1, \\
\left.\tilde{\Gamma}_{b u l k, r e n}^{(2,1)}(\mathbf{q}, \mathbf{Q} ; u, m)\right|_{\mathbf{q}=\mathbf{Q}=\mathbf{0}}=1, \\
\left.\tilde{\Gamma}_{b u l k, r e n}^{(4)}\left(\left\{\mathbf{q}_{i}\right\} ; u, m\right)\right|_{\left\{\mathbf{q}_{i}=\mathbf{0}\right\}}=m^{\epsilon} u
\end{gathered}
$$


for the renormalized vertex functions

$$
\Gamma_{b u l k, r e n}^{(N, I)}(\{\mathbf{q}, \mathbf{Q}\} ; m, u)=\left[Z_{\phi}(u)\right]^{N / 2}\left[Z_{\phi^{2}}(u)\right]^{I} \Gamma_{b u l k}^{(N, I)}\left(\{\mathbf{q}, \mathbf{Q}\} ; m_{0}^{2}, u_{0}\right) .
$$

Since the mass shift is sufficient to absorb the uv singularities of the bare functions $\Gamma_{b u l k}^{(N, I)}$, they become uv-finite when expressed in terms of $m$ and $u$ (or $m$ and $u_{0}$ ). The bulk renormalization factors can be written as

$$
\begin{gathered}
{\left[Z_{\phi}(u)\right]^{-1}=\left.\frac{\partial}{\partial q^{2}} \tilde{\Gamma}_{b u l k}^{(2)}\left[\mathbf{q} ; m_{0}^{2}(m, u), u_{0}(m, u)\right]\right|_{\mathbf{q}=\mathbf{0}},} \\
{\left[Z_{\phi^{2}} Z_{\phi}(u)\right]^{-1}=\tilde{\Gamma}_{b u l k}^{(2,1)}\left[\{\mathbf{0}\} ; m_{0}^{2}(m, u), u_{0}(m, u)\right],}
\end{gathered}
$$

and

$$
\left[Z_{u} Z_{\phi}^{2}(u)\right]^{-1}=\tilde{\Gamma}_{b u l k}^{(4)}\left[\{\mathbf{0}\} ; m_{0}^{2}(m, u), u_{0}(m, u)\right] / u_{0}(m, u) .
$$

\subsection{Surface normalization conditions}

Consider now the cumulants $G^{(N, M)}$ and $G^{\left(N, M ; I, I_{1}\right)}$ of our semi-infinite $\phi^{4}$ model. Let us stress that a surface-enhancement shift $\delta c$ is required to absorb uv singularities located on the surface. We write

$$
c_{0}=c+\delta c,
$$

where $c$ is a renormalized surface enhancement whose precise definition we still have to give.

We also know that the surface operators $\left.\phi_{s} \equiv \phi\right|_{z=0}$ and $\left(\phi_{s}\right)^{2}$ should scale with scaling dimensions that are different from those of their bulk analogs $\phi(\mathbf{x})$ and $\phi(\mathbf{x})^{2}$ with $\mathbf{x} \notin \partial V$. This suggests the introduction of separate renormalization factors for these surface operators, which we do via the relations

$$
\phi_{s}=\left[Z_{\phi} Z_{1}\right]^{1 / 2}\left[\phi_{s}\right]_{r e n}, \quad\left(\phi_{s}\right)^{2}=\left[Z_{\phi_{s}^{2}}\right]^{-1}\left[\left(\phi_{s}\right)^{2}\right]_{r e n}
$$

between the bare and renormalized operators. For the renormalized cumulants we thus have

$$
G_{r e n}^{(N, M)}(; m, u, c)=Z_{\phi}^{-(N+M) / 2} Z_{1}^{-M / 2} G^{(N, M)}\left(; m_{0}, u_{0}, c_{0}\right)
$$

and

$$
\begin{aligned}
& G_{r e n}^{\left(N, M ; I, I_{1}\right)}(; m, u, c) \\
& \quad=Z_{\phi}^{-(N+M) / 2} Z_{1}^{-M / 2}\left[Z_{\phi^{2}}\right]^{I}\left[Z_{\phi_{s}^{2}}\right]^{I_{1}} G^{\left(N, M ; I, I_{1}\right)}\left(; m_{0}, u_{0}, c_{0}\right) .
\end{aligned}
$$

We wish to fix $\delta c$ and the new renormalization factors $Z_{1}$ and $Z_{\phi_{s}^{2}}$ by appropriate normalization conditions. To motivate our choice, let us recall the perturbation expansion of the momentum-dependent surface susceptibility $\chi_{11}(\mathbf{p})=\hat{G}^{(0,2)}(\mathbf{p})$ to lowest order:

$$
\hat{G}^{(0,2)}\left(\mathbf{p} ; m_{0}, u_{0}, c_{0}\right)=\frac{1}{c_{0}+\sqrt{\mathbf{p}^{2}+m_{0}^{2}}}+\mathcal{O}\left(u_{0}\right) .
$$


We choose the normalization conditions such that the associated renormalized susceptibility and its first derivatives with respect to $p^{2}$ agree at $\mathbf{p}=\mathbf{0}$ with the corresponding zero-loop expressions implied by $(22)$, except for the replacements $m_{0} \rightarrow m$ and $c_{0} \rightarrow c$. This gives

$$
\left.\hat{G}_{r e n}^{(0,2)}(\mathbf{p} ; m, u, c)\right|_{\mathbf{p}=\mathbf{0}}=\frac{1}{m+c}
$$

and

$$
\left.\frac{\partial}{\partial p^{2}} \hat{G}_{r e n}^{(0,2)}(\mathbf{p} ; m, u, c)\right|_{\mathbf{p}=\mathbf{0}}=-\frac{1}{2 m(m+c)^{2}} .
$$

The following normalization condition fixes the correlation function with the insertion of the surface operator $\frac{1}{/} 2 \phi_{s}^{2}$, at zero external momenta:

$$
\left.\hat{G}_{r e n}^{(0,2 ; 0,1)}(\{\mathbf{p}\} ; m, u, c)\right|_{\{\mathbf{p}=\mathbf{0}\}}=(m+c)^{-2} .
$$

This choice is motivated by the definition of the bare function

$$
\hat{G}^{(0,2 ; 0,1)}(\{\mathbf{0}\})=-\frac{\partial}{\partial c_{0}} \hat{G}^{(0,2)}(\mathbf{0})
$$

whose perturbation expansion starts with $\left(m_{0}+c_{0}\right)^{-2}+\mathcal{O}\left(u_{0}\right)$.

Equation (23) defines the required surface-enhancement shift $\delta c$. It ensures, together with equation (10), that the special point is located at $m=c=0$. This equation implies also finite surface susceptibility at the ordinary transition when $m \rightarrow 0$ at fixed $c>0$. Equations (24) and (25) specify the renormalization factors $Z_{1}$ and $Z_{\phi_{s}^{2}}$ respectively, in a similar way as the bulk normalization conditions (11) and (12) define $Z_{\phi}$ and $Z_{\phi^{2}}$. The corresponding explicit expressions are

$$
Z_{1} Z_{\phi}=-\left.2 m(m+c)^{2} \frac{\partial}{\partial p^{2}} \hat{G}^{(0,2)}\left[\mathbf{p} ; m_{0}(m, u), u_{0}(m, u), c_{0}(c, m, u)\right]\right|_{\mathbf{p}=\mathbf{0}}
$$

and

$$
Z_{\phi_{s}^{2}}^{-1}=-\left.\left[Z_{1} Z_{\phi}\right]^{-1}(m+c)^{2} \frac{\partial}{\partial c_{0}} \hat{G}^{(0,2)}\left[\mathbf{0} ; m_{0}(m, u), u_{0}(m, u), c_{0}\right]\right|_{c_{0}=c_{0}(c, m, u)}
$$

The both above sets of normalization conditions define $m_{0}^{2}, u_{0}, Z_{\phi}, Z_{\phi^{2}}$, $c_{0}, Z_{1}$, and $Z_{\phi_{s}^{2}}$ as functions of $m, u, c$, and $\Lambda$. All $Z$-factors have the finite $\Lambda \rightarrow \infty$ limits in the $d<4$ case considered here.

Let us consider the $\Lambda=\infty$ limit in the sequel. In our calculations pertaining to the surface critical exponents we actually took $\Lambda=\infty$ from the outset, employing dimensional regularization. In this limit the bulk $Z$ factors $Z_{\phi}$ and $Z_{\phi^{2}}$ become functions of the single dimensionless variable $u$. However, with our choice of normalization conditions at the boundary, the surface $Z$ factors $Z_{1}$ and $Z_{\phi_{s}^{2}}$ depend both on $u$ and the dimensionless ratio $c / m$.

It would be essential to carry along the general dependence on the variable $c / m$ in an eventual investigation of the full crossover between different types of surface critical behaviour (see section 2). Our main objectve will be, however, the calculation of the surface critical exponents of the special transition. In order to do this it is sufficient to study the critical behaviour in the limit of $c / m \ll 1$. Therefore we focus directly on this asymptotic behaviour in the following. 


\section{Special Transition: general considerations}

Let us turn directly to the case of the special transition. In order to reach the corresponding multicritical point we can safely set $c=0$. This does not cause any problems in the theory, whenever the surface-enhancement renormalization has already been performed. We investigate then the corresponding critical behaviour by studying the theory at small temperature deviations from the bulk critical point $T_{c}$. Thus we obtain an "effective" massive theory, appropriate for the special transition, expressed in terms of the dimensionless bulk renormalized coupling constant $u$. The multicritical point is then reached in the theory as $u=u^{*}$, with $u^{*}$ the usual bulk fixed-point value of $u$. This way to access the description of the critical behaviour at the special transition in three dimensions (being valid for all space dimensions between $d_{*}(n)$ and $\left.d^{*}\right)$ seems us to be the simplest one. In the language of the bare theory this would correspond to working directly on the line $c_{0}=c_{0}^{s p}$ of the phase diagram.

\subsection{Normalization conditions}

At $c=0$ the surface normalization conditions are simplified and yield the value $c_{0}^{s p}=c_{0}^{s p}(u)$ and the surface renormalization $Z$ factors appropriate to the special transition:

$$
\begin{aligned}
& Z_{1}^{s p}(u) Z_{\phi}(u)=-\left.2 m^{3} \frac{\partial}{\partial p^{2}} \hat{G}^{(0,2)}\left[p ; m_{0}(m, u), u_{0}(m, u), c_{0}^{s p}(u)\right]\right|_{p=0}, \quad(29) \\
& {\left[Z_{\phi_{s}^{2}}^{s p}(u)\right]^{-1}=-\left.m^{2}\left[Z_{1}^{s p} Z_{\phi}\right]^{-1} \frac{\partial}{\partial c_{0}} \hat{G}^{(0,2)}\left[0 ; m_{0}(m, u), u_{0}(m, u), c_{0}\right]\right|_{c_{0}=c_{0}^{s p}(u)}}
\end{aligned}
$$

The above-defined surface renormalization factors enter the corresponding relations (20) between the bare and renormalized correlation functions at the line $c=0$ :

$$
G_{r e n, s p}^{(N, M)}(; m, u)=Z_{\phi}^{-(N+M) / 2}\left(Z_{1}^{s p}\right)^{-M / 2} G^{(N, M)}\left(; m_{0}, u_{0}, c_{0}^{s p}\right) .
$$

\subsection{Callan-Symanzik-type equations}

By varying $m$ at fixed $u_{0}$ and $c_{0}^{s p}$ the analogs of the Callan-Symanzik equation (see references $[3,19,20]$ ) for the correlation functions $G_{r e n, s p}^{(N, M)}$ can be derived in a straightforward fashion:

$$
\left[m \frac{\partial}{\partial m}+\beta(u) \frac{\partial}{\partial u}+\frac{N+M}{2} \eta_{\phi}(u)+\frac{M}{2} \eta_{1}^{s p}(u)\right] G_{r e n, s p}^{(N, M)}(; m, u)=\Delta G
$$

with

$$
\Delta G \equiv-\left[2-\eta_{\phi}(u)\right] m^{2} \int_{V} d^{d} X G_{r e n, s p}^{(N, M ; 1,0)}(; m, u),
$$

where the integration is over the position $\mathbf{X}$ of the inserted $\phi^{2}$ operator.

Just as in the bulk case, and as could be corroborated by means of a short-distance expansion, the inhomogeneity $\Delta G$ on the right-hand side should be negligible in the critical regime. The resulting homogeneous equation differs from the standard bulk Callan-Symanzik equation mainly in that it involves the new function $\eta_{1}^{s p}(u)$, and can be integrated in a standard fashion. 
The coefficient functions in the equation (32) are:

$$
\begin{gathered}
\beta(u)=\left.m \frac{\partial}{\partial m}\right|_{0} u \\
\eta_{\phi}(u)=\left.m \frac{\partial}{\partial m}\right|_{0} \ln Z_{\phi}(u)=\beta(u) \frac{d \ln Z_{\phi}(u)}{d u}
\end{gathered}
$$

which are the standard bulk ones, and

$$
\eta_{1}^{s p}(u)=\left.m \frac{\partial}{\partial m}\right|_{0} \ln Z_{1}^{s p}(u)=\beta(u) \frac{d \ln Z_{1}^{s p}(u)}{d u}
$$

which is specific for our semi-infinite system. The symbol $\left.\right|_{0}$ indicates that the derivatives are taken at fixed bare coupling constant and surface enhancement (and cutoff $\Lambda$ ).

\section{3. $\quad Z$ factors of the massive theory and scaling at the multicritical point}

In contrast to the similar formulas of the massless theory conventionally used in the investigations of semi-infinite systems [15] and containing an arbitrary normalization scale $\mu$ with no direct physical meaning, here, as usual in the massive theory $[3,19,20]$, the variable $m$ is identified with the inverse bulk correlation length $\xi^{-1}$ and thus, is the singular function of the temperature:

$$
m=\xi^{-1} \propto t^{\nu}, \quad t \propto\left(T-T_{c}\right) / T_{c} .
$$

Furter, in the vicinity of the infrared-stable zero $u^{*}$ of the $\beta$ function $\left[\beta^{\prime}\left(u^{*}\right) \equiv \omega>0\right]$ the property holds

$$
\left|u-u^{*}\right| \propto m^{\omega},
$$

and the singular behaviour of the renormalization $Z$ factors in the critical domain is

$$
\begin{aligned}
Z_{\phi} & \propto m^{\eta}, \\
Z_{1}^{s p} & \propto m^{\eta_{1}^{s^{s}}}
\end{aligned}
$$

where $\eta=\eta\left(u^{*}\right)$ is the standard bulk correlation exponent, and $\eta_{1}^{s p}=$ $\eta_{1}^{s p}\left(u^{*}\right)$.

Let us now turn back to equation (31) relating the bare and renormalized correlation functions at $c_{0}=c_{0}^{s p}(c=0)$. We take for transparency the simple case $N=0, M=2$ and consider the parallel Fourier transform of the bare function $G^{(0,2)}\left(r ; m_{0}, u_{0}, c_{0}^{s p}\right)$ :

$$
\hat{G}^{(0,2)}\left(p ; m_{0}, u_{0}, c_{0}^{s p}\right)=Z_{\phi} Z_{1}^{s p} \hat{G}_{r e n, s p}^{(0,2)}(p ; m, u) .
$$

Using the simple dimensional analysis for $\hat{G}_{r e n, s p}^{(0,2)}$ and the mass dependences (39) and (40) of $Z$ factors near the critical point we get

$$
\begin{aligned}
\hat{G}^{(0,2)}\left(p ; m_{0}, u_{0}, c_{0}^{s p}\right) & \propto m^{-1+\eta+\eta_{1}^{s p}} \hat{G}_{r e n, s p}^{(0,2)}\left(\frac{p}{m} ; 1, u^{*}\right) \\
& \propto t^{-\nu\left(1-\eta-\eta_{1}^{s p}\right)} G^{(0,2)}\left(p t^{-\nu}\right) .
\end{aligned}
$$


This is the scaling form for the pair correlation function of the boundary fields at the special transition which identifies (see [15]) the surface susceptibility exponent

$$
\gamma_{11}=\nu\left(1-\eta-\eta_{1}^{s p}\right)
$$

and the surface correlation exponent

$$
\eta_{\|}^{s p}=\eta_{1}^{s p}+\eta
$$

\subsection{Expansion about the multicritical point; identification of $\Phi$}

In order to complete our picture of the special transition we have to identify the remaining crossover exponent $\Phi$. This requires considerations in the limit of small $c\left(c_{0}\right.$ in the vicinity of $\left.c_{0}^{s p}\right)$, rather than at $c=0\left(c_{0}=c_{0}^{s p}\right)$. In doing so, we can expand the bare correlation functions $G^{(N, M)}\left(; m_{0}, u_{0}, c_{0}\right)$ in powers of the small deviation $\delta c_{0} \equiv c_{0}-c_{0}^{s p}$ :

$$
G^{(N, M)}\left(; m_{0}, u_{0}, c_{0}\right)=\sum_{K \geqslant 0} \frac{1}{K !} G^{(N, M ; 0, K)}\left(; m_{0}, u_{0}, c_{0}^{s p}\right)\left(\delta c_{0}\right)^{K} .
$$

In our expansion no danger of the infrared divergences occurs like in the conventional small- $t$ expansion near the critical point in the bulk (see e.g. $[3,36]$ ) which we follow closely, since we perform it in the massive theory, remaining outside of the critical temperature. We reexpress now the righthand side of the last equation in terms of the renormalized correlation functions according to the theory defined previously for the special point $c=0$ and obtain the relation

$$
\begin{aligned}
& Z_{\phi}^{-(N+M) / 2}\left(Z_{1}^{s p}\right)^{-M / 2} G^{(N, M)}\left(; m_{0}, u_{0}, c_{0}\right) \\
= & \sum_{K \geqslant 0} \frac{1}{K !} G_{r e n, s p}^{(N, M ; 0, K)}(; m, u)\left[\left(Z_{\phi_{s}^{2}}^{s p}\right)^{-1} \delta c_{0}\right]^{K} .
\end{aligned}
$$

Here, for the finite renormalized deviation $\delta c$ of the surface enhancement from its special value $c=0$,

$$
\delta c \equiv\left[Z_{\phi_{s}^{2}}^{s p}(u)\right]^{-1} \delta c_{0},
$$

we obtain the finite renormalized correlation function

$$
G_{r e n}^{(N, M)}(; m, u, \delta c)=Z_{\phi}^{-(N+M) / 2}\left(Z_{1}^{s p}\right)^{-M / 2} G^{(N, M)}\left(; m_{0}, u_{0}, c_{0}\right)
$$

defined in the vicinity of the multicritical point and related to its bare counterpart through the renormalization $Z$ factors defined at the special point $c=0$.

For dimensional reasons, the renormalized correlation functions actually depend in the neighbourhood of the special point on the dimensionless variable

$$
\mathrm{c} \equiv \delta c / m \text {. }
$$

Owing to the presence of this variable, the Callan-Symanzik equation for the correlation function $G_{r e n}^{(N, M)}(; m, u, c)$ (cf. equation (32)) will contain an additional differential operator

$$
-\left[1+\eta_{c}^{s p}(u)\right] \mathrm{c} \frac{\partial}{\partial \mathrm{c}} .
$$


It is of the same form as in references $[37,15]$ and includes the Wilson function

$$
\eta_{c}^{s p}(u)=\left.m \frac{\partial}{\partial m}\right|_{0} \ln Z_{\phi_{s}^{2}}^{s p}(u)=\beta(u) \frac{d \ln Z_{\phi_{s}^{2}}^{s p}(u)}{d u} .
$$

As before, the renormalization factor $Z_{\phi_{s}^{2}}^{s p}$ behaves as

$$
Z_{\phi_{s}^{2}}^{s p} \propto m^{\eta_{c}^{s p}}
$$

near the critical temperature, with $\eta_{c}^{s p}=\eta_{c}^{s p}\left(u^{*}\right)$. This implies, together with the equation (47), the non-analytic temperature dependence of the renormalized surface-enhancement deviation from the special point through the relation

$$
\delta c \propto m^{-\eta_{c}^{s p}} \delta c_{0} .
$$

For the surface-fields correlation function $\hat{G}^{(0,2)}(p)$ we obtain thus, in the neighbourhood of the multicritical point, the scaling form

$$
\begin{aligned}
\hat{G}^{(0,2)}\left(p ; m_{0}, u_{0}, c_{0}\right) & \propto m^{-\frac{\gamma_{11}}{\nu}} \hat{G}_{r e n}^{(0,2)}\left(\frac{p}{m} ; 1, u^{*}, \frac{\delta c}{m}\right) \\
& \propto t^{-\gamma_{11}} G^{(0,2)}\left[p t^{-\nu} ; \delta c_{0} t^{-\nu\left(1+\eta_{c}^{s p}\right)}\right] .
\end{aligned}
$$

Hence, the surface crossover critical exponent $\Phi$ is identified (see [15]),

$$
\Phi=\nu\left(1+\eta_{c}^{s p}\right) .
$$

\section{Perturbation theory}

Let us consider now the explicit renormalization-group factors $Z_{1}^{s p}, Z_{\phi_{s}^{2}}^{s p}$, and related $\eta$ functions. To the one-loop order, the calculation is carried out for general dimensions $d<4$. The two-loop results are restricted to the case $d=3$.

\subsection{General}

The free propagator of our perturbation theory was already given in equation (6). We start with the diagrammatic expansion of the simplest surface connected correlation function $\hat{G}^{(0,2)}\left(p ; m_{0}, u_{0}, c_{0}\right)$,

$$
\hat{G}^{(0,2)}\left(p ; m_{0}, u_{0}, c_{0}\right)=\hat{G}\left(p ; z=z^{\prime}=0 ; c_{0}\right)+\ldots
$$

where the zero-order contribution is given by the free propagator (6) "at the surface", $\left(\kappa_{0}+c_{0}\right)^{-1}$. "+..." means the presence of the usual connected Feynman graphs of the $\phi^{4}$ theory with two external legs. Beyond zeroth order, in subsequent terms of $\hat{G}^{(0,2)}(p)$ each external line joins a point at the surface ( say, $z^{\prime}=0$ ) with another arbitrary point $z \geqslant 0$ of the system. The corresponding external propagator is

$$
\hat{G}\left(p ; z, 0 ; c_{0}\right)=\frac{e^{-\kappa_{0} z}}{\kappa_{0}+c_{0}} .
$$


We note that in order to facilitate the treatment of external lines depending both on the external momentum and the surface enhancement, it is useful to "amputate" denominators $\kappa_{0}+c_{0}$ from each external propagator. This is achieved, in analogy with the infinite-volume theory, by inverting the series expansion (56). Thus, we consider (to the perturbation-theory order we are interested in)

$$
\hat{G}^{(0,2)-1}\left(p ; m_{0}, u_{0}, c_{0}\right)=\kappa_{0}+c_{0}-G_{1}(p)-\Sigma_{2}(p)+\left(\kappa_{0}+c_{0}\right)^{-1}\left[G_{1}(p)\right]^{2}
$$

where $G_{1}(p)$ is the first-order term (a single loop) and $\Sigma_{2}(p)$ represents the sum of the second-order contributions,

$$
\Sigma_{2}(p)=G_{2}(p)+G_{3}(p)+G_{4}(p) .
$$

$G_{i}(p)$ are the contributions of the two-loop Feynman diagrams (i):

(2) "melon" graph with three equivalent internal lines;

(3) one-particle reducible graph: a line joins two single loops;

(4) one-particle irreducible graph: a loop at the top of another loop.

The "amputated" external propagators in these graphs reduce to $e^{-\kappa_{0} z}$. Internal lines remained unchanged, and denote the full free propagators (6). Unlike the procedure of inverting the connected pair correlation function $\tilde{G}_{b u l k}^{(2 ; 0)}$ in the bulk theory yielding the amputated, one-particle-irreducible vertex function $\tilde{\Gamma}_{b u l k}^{(2 ; 0)}$, here, as a consequence of the absence of translational invariance along the $z$ direction, only a partial cancellation in the external legs occurs, and the one-line-reducible graphs do not disappear.

We find it to be convenient to calculate using the inverse surface correlation function $\left[\hat{G}^{(0,2)}\right]^{-1}$, and thus, we would like to re-express several above formulas in terms of it. First, let us simply write the normalization condition (23) in terms of the inverse bare correlation function:

$$
Z_{1} Z_{\phi} \hat{G}^{(0,2)-1}\left(0 ; m_{0}, u_{0}, c_{0}\right)=m+c .
$$

Next, we transform the momentum derivative in the formula (27) for $Z_{1} Z_{\phi}$ and making use of the normalization (60) obtain

$$
\left(Z_{1} Z_{\phi}\right)^{-1}=\left.2 m \frac{\partial}{\partial p^{2}}\left[\hat{G}^{(0,2)}\left(p^{2}\right)\right]^{-1}\right|_{p^{2}=0}=\lim _{p \rightarrow 0} \frac{m}{p} \frac{\partial}{\partial p}\left[\hat{G}^{(0,2)}(p)\right]^{-1} .
$$

Finally, in a similar way we re-write also the expression (28) for the $Z$ factor $Z_{\phi_{s}^{2}}$ :

$$
Z_{\phi_{s}^{2}}^{-1}=Z_{1} Z_{\phi} \frac{\partial}{\partial c_{0}}\left[\hat{G}^{(0,2)}(0)\right]^{-1} .
$$

\subsection{One-loop approximation}

Let us calculate the renormalization factor $Z_{1}^{s p} Z_{\phi}$ to the first order of the perturbation theory, employing the definition (61). We start from the one-loop part of the expansion of the inverse surface correlation function $\hat{G}^{(0,2)-1}\left(p ; m_{0}, u_{0}, c_{0}\right)$, equation (58). The mass renormalization (via equation (10)) therein cancels the divergent part of the one-loop contribution associated with the translationally-invariant, bulk part of the free propagator (6). Since we are currently interested only in the momentum-dependent 
terms, we are allowed in the present calculation to simply replace the bare surface enhancement $c_{0}$ by its renormalized counterpart $c$ and set it equal to zero. Hence the one-loop renormalization factor $\left(Z_{1}^{s p} Z_{\phi}\right)^{-1}$ is given by

$$
\left(Z_{1}^{s p} Z_{\phi}\right)^{-1}=\left(Z_{1}^{s p}\right)^{-1}=1-\left.\frac{\partial}{\partial \kappa} G_{1}^{(+)}(p)\right|_{\kappa=m} .
$$

We omit the factor $Z_{\phi}$ here since it produces only the second-order and higher corrections. The superscript + at $G_{1}$ indicates that the internal line of this graph associates now with the propagator

$$
\hat{G}_{+}\left(p ; z, z^{\prime}\right)=\frac{1}{2 \kappa} e^{-\kappa\left(z+z^{\prime}\right)}
$$

which is the "surface" part of the free propagator (6). The explicit expression for the relevant one-loop graph reads

$$
G_{1}^{(+)}(p)=-\frac{n+2}{6} u_{0} \int_{0}^{\infty} d z e^{-2 \kappa z} \int_{\mathbf{p}_{1}} \frac{e^{-2 \kappa_{1} z}}{2 \kappa_{1}}
$$

with $\kappa=\sqrt{p^{2}+m^{2}}$ and $\kappa_{1}=\sqrt{p_{1}^{2}+m^{2}}$.

We calculate $Z_{1}^{s p}$ in the dimensionally regularized form using the mathematical tables [38]. As usual, the factor $m^{-\epsilon}$ appears, multiplying the bare coupling constant $u_{0}$. Hence, the dimensionless effective expansion parameter arises, $\bar{u}_{0}=u_{0} m^{-\epsilon}$. To the present order, $\bar{u}_{0}$ is identified, through the vertex renormalization (13), with the renormalized coupling constant $u$. Our result is

$$
Z_{1}^{s p}=1+\frac{n+2}{6} u \frac{1}{1+\epsilon} 2^{-d} \pi^{-\frac{d}{2}} \Gamma\left(\frac{\epsilon}{2}\right) .
$$

Similar calculation of the next renormalization factor $Z_{\phi_{s}^{2}}^{s p}$ through equation (62) yields

$$
Z_{\phi_{s}^{2}}^{s p}=1-\frac{n+2}{6} u \frac{\pi^{-\frac{d-1}{2}}}{16} \frac{\Gamma(\epsilon)}{\Gamma\left(\frac{3+\epsilon}{2}\right)}\left[1-2^{\frac{1+\epsilon}{2}}{ }_{2} F_{1}\left(\frac{3-\epsilon}{2}, \frac{1+\epsilon}{2} ; \frac{3+\epsilon}{2} ; \frac{1}{2}\right)\right] .
$$

Here ${ }_{2} F_{1}(\ldots)$ is the hypergeometric function [39].

In our massive theory the value of $\epsilon$ is not necessarily restricted to be infinitesimally small and the above expressions hold for any relevant dimensions $d<4$ (see Section 2). The first-order coefficients at $u$ are universal in the same sense as the two first $\beta$ function coefficients [3], being independent of the renormalization scheme.

The presence of the Euler gamma function $\Gamma(\epsilon)$ indicates the dimensional poles $1 / \epsilon$ when $d$ approaches to 4 . In this limit, corresponding to the $\epsilon$ expansion, equations (66) and (67) reduce to

$$
Z_{1(\epsilon \rightarrow 0)}^{s p}=Z_{\phi_{s}^{2}(\epsilon \rightarrow 0)}^{s p}=1+\frac{n+2}{6 \epsilon} \frac{u}{8 \pi^{2}}
$$

This agrees with the one-loop result [37] obtained in the massless theory employing the minimal subtraction scheme ${ }^{3}$. In another special case, $d=3$,

\footnotetext{
${ }^{3}$ Recall that in contrast to the present approach, the dimensionless coupling constant $u$ is introduced in that scheme by absorbing the dimensional factor $\mu^{-\epsilon}, \mu$ being an arbitrary momentum subtraction point.
} 
the renormalization factors $Z_{1}^{s p}$ and $Z_{\phi_{s}^{2}}^{s p}$ are completely finite, and their oneloop expressions do not coincide. They can be read-off from the two-loop formulas given in the next subsection ${ }^{4}$.

The procedure of the computation of the associated exponent Wilson functions $\eta_{1}^{s p}(u)$ and $\eta_{c}^{s p}(u)$ through equations (36) and (51) eliminates the poles at $d \rightarrow 4$ yielding the expressions finite up to $d=4$ :

$$
\eta_{1}^{s p}(u)=-\frac{n+2}{6} u \frac{\epsilon}{1+\epsilon} 2^{-d} \pi^{-\frac{d}{2}} \Gamma\left(\frac{\epsilon}{2}\right)
$$

and

$$
\eta_{c}^{s p}(u)=\frac{n+2}{6} \frac{u \epsilon}{1+\epsilon} 2^{-d} \pi^{-\frac{d}{2}} \Gamma\left(\frac{\epsilon}{2}\right)\left[1-2^{\frac{1+\epsilon}{2}}{ }_{2} F_{1}\left(\frac{3-\epsilon}{2}, \frac{1+\epsilon}{2} ; \frac{3+\epsilon}{2} ; \frac{1}{2}\right)\right] .
$$

Their leading terms of the $\epsilon$ expansion

$$
\eta_{1(\epsilon \rightarrow 0)}^{s p}=\eta_{c(\epsilon \rightarrow 0)}^{s p}=-\frac{n+2}{6} \frac{u}{8 \pi^{2}}
$$

agree with the known one-loop results $[37,15]$. The corresponding expressions for $d=3$ are contained in the two-loop formulas given below.

Let us evaluate our $\eta$ functions at the fixed point $u=u^{*}$ which is the infrared-stable zero of the $\beta$ function (see e.g. references $[35,36,3]$ ). In the present approximation, the one-loop $\beta$ function yields

$$
u^{*}=\frac{6}{n+8} 2^{d} \pi^{\frac{d}{2}} \frac{1}{\Gamma\left(\frac{\epsilon}{2}\right)} .
$$

Substituting this in equations (69), (70) we obtain

$$
\begin{aligned}
\eta_{1}^{s p}\left(u^{*}\right) & =-\frac{n+2}{n+8} \frac{\epsilon}{1+\epsilon} \\
\eta_{c}^{s p}\left(u^{*}\right) & =\frac{n+2}{n+8} \frac{\epsilon}{1+\epsilon}\left[1-2^{\frac{1+\epsilon}{2}}{ }_{2} F_{1}\left(\frac{3-\epsilon}{2}, \frac{1+\epsilon}{2} ; \frac{3+\epsilon}{2} ; \frac{1}{2}\right)\right] .
\end{aligned}
$$

Let us recall once more that the present expressions are valid for arbitrary $\epsilon>0$ down to the lower critical dimension $d_{*}(n)$ (see section 2). As $\epsilon \rightarrow 0$ the well-known correct results (see references $[37,15]$ and references therein) are reproduced. At $d=3$ from the last formulas the lowest-order numerical estimates may be obtained

$$
\begin{aligned}
\eta_{1}^{s p} & =-\frac{1}{2} \frac{n+2}{n+8}, \\
\eta_{c}^{s p} & =-2 \frac{n+2}{n+8}\left(\ln 2-\frac{1}{4}\right) .
\end{aligned}
$$

It is amazing to recognize that the values $\eta_{1}^{s p}(n=0)=\eta_{\|}^{s p}(n=0)=$ $-1 / 8 \simeq-0.13$ and $\eta_{1}^{s p}(n=1)=\eta_{\|}^{s p}(n=1)=-1 / 6 \simeq-0.17$ obtained in this simple calculation lie just among the best estimates of $\eta_{\|}^{s p}$ resulting

\footnotetext{
${ }^{4}$ Note the different numerical normalizations of the renormalized coupling constant $u$ used here and in Subsection 5.3.
} 
from our involved two-loop calculations (see tables 1 and 2). This occurence might be understood in the context of the $\epsilon$ expansion. Our one-loop expression (73) for $\eta_{1}^{s p}\left(u^{*}\right)$ differs from the standard one-loop $\epsilon$-expansion result by the denominator $1+\epsilon$. This denominator represents, apparently, the result of certain partial resummation of the whole $\epsilon$ expansion of $\eta_{\|}^{s p}$ : this denominator, expanded in small $\epsilon$, would bring its contribution to each order of the true $\epsilon$ expansion. The role played by this denominator is the suppresion of the linear growth of $\eta_{\|}^{s p}$ with $\epsilon$. This is a right tendency, whose effect is similar, in the present situation, to the inclusion of the $\epsilon^{2}$ term in the true $\epsilon$ expansion. This improvement happened to be quite successful, as was illustrated just above ${ }^{5}$.

\subsection{Two-loop approximation}

In the next order of the perturbation theory we restrict ourselves to the computations at $d=3$. The details can be found elswhere [33], and here we only quote the final results. In terms of the renormalized coupling constant normalized as usual so that $\beta(u)=-u+u^{2}+O\left(u^{3}\right)$ we have obtained

$$
\begin{aligned}
Z_{1}^{s p} Z_{\phi}=1 & +\frac{n+2}{2(n+8)} u-\frac{12(n+2)}{(n+8)^{2}}\left[A-\frac{(n+2)}{12}(1-\ln 2) \ln 2-\frac{n+14}{48}\right] u^{2}, \\
Z_{\phi_{s}^{2}}^{s p} & =1+2 \frac{n+2}{n+8}\left(\ln 2-\frac{1}{4}\right) u \\
& +\frac{12(n+2)}{(n+8)^{2}}\left[A-B-\frac{n}{2} \ln 2+\frac{(n+2)}{2} \ln ^{2} 2+\frac{2 n+1}{12}\right] u^{2}
\end{aligned}
$$

and

$$
\begin{gathered}
\eta_{\|}^{s p}(u)=-\frac{n+2}{2(n+8)} u+\frac{12(n+2)}{(n+8)^{2}}\left[2 A-\frac{n+2}{6}(1-\ln 2) \ln 2+\frac{n-10}{48}\right] u^{2}, \\
\eta_{c}^{s p}(u)=-2 \frac{n+2}{n+8}\left(\ln 2-\frac{1}{4}\right) u \\
-\frac{12(n+2)}{(n+8)^{2}}\left[2(A-B)-(n+1) \ln 2+2 \frac{n+2}{3} \ln ^{2} 2+\frac{17 n+22}{48}\right] u^{2}
\end{gathered}
$$

The numbers $A$ and $B$ are associated with contributions of the two-loop diagram with three equivalent internal lines. We have determined them by numerical integration, obtaining $A=0.202428$ and $B=0.678061$ (see reference [33]).

Analogous series expansions for the other surface exponents can be derived from the present results by means of the scaling laws.

\footnotetext{
${ }^{5}$ It is well-known that the numerical values of many critical exponents resulting from the second-order $\epsilon$ expansion at $\epsilon=1$ and from the second-order massive-theory calculations in three dimensions are rather close one to another and agree well with the best estimates of these exponents.
} 


\section{Critical exponents}

In this section we shall discuss the numerical values of the surface critical exponents which can be extracted from the above results. Let us quote here, for the sake of completeness, the list of scaling relations [15] which were used to generate the additional perturbation-theory expansions:

$$
\begin{aligned}
\Delta_{1} & =\frac{\nu}{2}\left(d-\eta_{\|}\right) \\
\eta_{\perp} & =\frac{1}{2}\left(\eta+\eta_{\|}\right) \\
\beta_{1} & =\frac{\nu}{2}\left(d-2+\eta_{\|}\right) \\
\gamma_{11} & =\nu\left(1-\eta_{\|}\right) \\
\gamma_{1} & =\nu\left(2-\eta_{\perp}\right) \\
\delta_{1} & =\frac{\Delta}{\beta_{1}}=\frac{d+2-\eta}{d-2+\eta_{\|}} \\
\delta_{11} & =\frac{\Delta_{1}}{\beta_{1}}=\frac{d-\eta_{\|}}{d-2+\eta_{\|}} \\
\alpha_{1} & =\alpha+\nu-1+\Phi \\
\alpha_{11} & =\alpha+\nu-2+2 \Phi .
\end{aligned}
$$

Here $\Delta_{1}$ is the surface magnetic shift exponent; $\eta_{\perp}$ characterizes criticalpoint correlations in $z$ direction perpendicular to the surface; $\beta_{1}$ characterizes the decay of the surface magnetization on approaching the critical temperature; $\gamma_{11}$ is the (local) surface susceptibility exponent; $\gamma_{1}$ is the layer susceptibility exponent; $\delta_{11}$ and $\delta_{1}$ express the relation of the surface magnetization to the surface and bulk external magnetic field, respectively, along the critical isotherm; $\alpha_{1}$ and $\alpha_{11}$ are the exponents of layer and local specific heats.

Above scaling relations are used at $d=3$. They contain bulk correlation length critical exponent $\nu$, critical-point pair correlation function exponent $\eta$ and bulk shift exponent $\Delta=\frac{1}{2} \nu(d+2-\eta)$. To the needed perturbationtheory order, the series expansions for $\nu$ and $\eta$ are given in three dimensions by

$$
\begin{aligned}
\nu & =\frac{1}{2}\left[1+\frac{1}{2} \frac{n+2}{n+8} u+\frac{n+2}{(n+8)^{2}} \frac{27 n-38}{108} u^{2}\right] \\
\eta & =\frac{8}{27} \frac{n+2}{(n+8)^{2}} u^{2} .
\end{aligned}
$$

Power series expansions of this kind are in general divergent: their partial sums of increasing order do not converge to the actual sum of the series. This in principle prevents approximating the physical constant's value represented by its truncated series expansion in $u$ by simply substituting there $u=u^{*}$. The appropriate approach is to "resum" series like (79), (80). The simplest procedure is to construct the table of Padé approximants [40]. This works well when the series behave in lowest orders "in a convergent fashion". Apparently divergent ones require more sophisticated summation 
procedures. The simplest is the Padé-Borel method of reference [41]. To the present, second order of perturbation series, this method involves the analytic continuation of the Borel transform by [1/1] Padé approximant.

Our series expansions for surface critical exponents have the form $I=$ $c_{0}+c_{1} u+c_{2} u^{2}$. For each exponent we modified the expansion either into $I / c_{0}=1+\left(c_{1} / c_{0}\right) u+\left(c_{2} / c_{0}\right) u^{2}$ if $c_{0}>1$, or $I+\left(1-c_{0}\right)=1+c_{1} u+c_{2} u^{2}$ if $c_{0}<$ 1 , both starting by unity. Afterwards we returned to the desired exponent by inverting these procedures in terms of the numerical values computed at $u=u^{*}$. This allowed us to see easily the relative values of subsequent orders, and to consider preferably the expansions with possibly smallest corrections to the leading order. If one uses the second procedure for the truncated expansion instead of simply factorize its $c_{0}<1$, one obtains the better convergence of the corresponding inverse expansion. The rate of this "convergence" is controlled by the ratios $O_{1} / O_{2}$ of the first- and second-order corrections for direct $\left(1+O_{1}+O_{2}\right)$ expansions, and $O_{1 i} / O_{2 i}$ for inverse $\left(1+O_{1 i}+O_{2 i}\right)$ expansions. These are given in the first row of our tables. The bigger is the absolute value of the ratio, the better is the quality of the series. Negative ratios correspond to altering in sign series segments. In these cases the Padé-Borel resummations may be performedђ. Positive ratios would correspond to the truncated series that do not alter in sign. These are not adapted for the Padé-Borel resummation since the [1/1] approximant of the Borel transform has the pole at the positive real axis, i.e. in the integration range (see reference [5]).

All the available series expansions in $u$ alter in sign. They are mainly slowly convergent. Exceptions are some series involving $\eta_{c}^{s p}$, whose behaviour is rather bad. The results of Padé analyses and Padé-Borel resummations are given in tables 1-4. Therein, $R$ indicates numerical estimates from the resummation of the direct series. $R_{i n v}^{-1}$ stands for the estimates coming from the resummations of the inverse series expansions. Let us recall that we used the hyperscaling relations with $d=3$. This is why our zeroth approximations do not always coincide with the classical Landau values of the critical exponents, which are the starting positions in the $\epsilon$ expansions. The latter are, in the same sequence as in the tables, $0,1,0$, $1 / 2,1 / 2,1,3,2[15,14]$.

To be consistent in the approximation's order, we used in our numerical estimations the fixed-point values $u^{*}$ from the Padé-Borel resummed twoloop $\beta$ functions $[41-43]$.

Let us consider the first group of exponents $\eta_{\|}, \ldots, \delta_{11}$ (tables 1 and 2) related to the function $\eta_{1}^{s p}(u)$.

Table 1. Special transition surface critical exponents associated to the renormalization-group function $\eta_{1} \cdot n=0, u^{*}=$ 1.632 .

\begin{tabular}{|c|c|l|l|l|l|l|l|l|l|c|}
\hline Index & $O_{1(i)} / O_{2(i)}$ & {$[0 / 0]$} & {$[1 / 0]$} & {$[0 / 1]$} & {$[2 / 0]$} & {$[0 / 2]$} & {$[1 / 1]$} & $R$ & $R_{i n v}^{-1}$ & $I\left(\Delta_{1}, \nu, \eta\right)$ \\
\hline$\eta_{\|}$ & $-1.6(-2.4)$ & 0.00 & -0.204 & 0.169 & -0.079 & -0.107 & -0.126 & -0.134 & 0.130 & -0.133 \\
$\Delta_{1}$ & $-4.8(-2.4)$ & 0.75 & 0.954 & 1.006 & 0.911 & 0.886 & 0.919 & 0.921 & 0.926 & 0.921 \\
$\eta_{\perp}$ & $-1.4(-1.2)$ & 0.00 & -0.102 & 0.114 & -0.027 & -0.017 & -0.059 & -0.063 & -0.064 & -0.053 \\
$\beta_{1}$ & $0.0(0.0)$ & 0.25 & 0.250 & 0.250 & 0.264 & 0.264 & - & - & - & 0.255 \\
$\gamma_{11}$ & $-3.6(-2.1)$ & 0.50 & 0.704 & 0.756 & 0.647 & 0.618 & 0.660 & 0.663 & 0.668 & 0.666 \\
$\gamma_{1}$ & $-4.5(-2.1)$ & 1.00 & 1.255 & 1.342 & 1.199 & 1.154 & 1.209 & 1.212 & 1.220 & 1.207 \\
$\delta_{1}$ & $-2.3(-1.6)$ & 5.00 & 6.020 & 6.281 & 5.576 & 5.397 & 5.711 & 5.739 & 5.764 & 5.734 \\
$\delta_{11}$ & $-2.4(-1.5)$ & 3.00 & 3.816 & 4.121 & 3.481 & 3.283 & 3.578 & 3.599 & 3.628 & 3.612 \\
\hline
\end{tabular}

The most reliable estimates are obtained here from the direct series for the exponent $\Delta_{1}$, which appear to exhibit the best convergence properties. 
Table 2. Special transition surface critical exponents associated to the renormalization-group function $\eta_{1} \cdot n=1, u^{*}=$ 1.597 .

\begin{tabular}{|c|c|l|l|l|l|l|l|l|l|c|}
\hline Index & $O_{1(i)} / O_{2(i)}$ & {$[0 / 0]$} & {$[1 / 0]$} & {$[0 / 1]$} & {$[2 / 0]$} & {$[0 / 2]$} & {$[1 / 1]$} & $R$ & $R_{\text {inv }}^{-1}$ & $I\left(\Delta_{1}, \nu, \eta\right)$ \\
\hline$\eta_{\|}$ & $-2.1(-4.8)$ & 0.00 & -0.266 & 0.210 & 0.140 & -0.174 & 0.181 & -0.189 & 0.183 & -0.165 \\
$\Delta_{1}$ & $-12.7(-2.9)$ & 0.75 & 1.016 & 1.113 & 0.995 & 0.961 & 0.997 & 0.997 & 1.006 & 0.997 \\
$\eta_{\perp}$ & $-1.7(-1.4)$ & 0.00 & 0.133 & 0.154 & -0.056 & -0.040 & -0.084 & -0.089 & -0.091 & -0.067 \\
$\beta_{1}$ & $0.0(0.0)$ & 0.25 & 0.250 & 0.250 & 0.261 & 0.262 & - & - & - & 0.263 \\
$\gamma_{11}$ & $-8.2(-2.6)$ & 0.50 & 0.766 & 0.863 & 0.734 & 0.695 & 0.737 & 0.739 & 0.747 & 0.734 \\
$\gamma_{1}$ & $-11.0(-2.4)$ & 1.00 & 1.333 & 1.499 & 1.302 & 1.237 & 1.305 & 1.306 & 1.320 & 1.302 \\
$\delta_{1}$ & $-4.4(-2.0)$ & 5.00 & 6.331 & 6.814 & 6.028 & 5.779 & 6.084 & 6.101 & 6.147 & 5.951 \\
$\delta_{11}$ & $-4.8(-1.8)$ & 3.00 & 4.065 & 4.650 & 3.845 & 3.553 & 3.882 & 3.894 & 3.947 & 3.791 \\
\hline
\end{tabular}

Table 3. Special transition surface critical exponents associated to the renormalization-group function $\eta_{c} . n=0, u^{*}=$ 1.632 .

\begin{tabular}{|c|l|l|l|l|l|l|l|l|l|c|}
\hline Index & $O_{1(i)} / O_{2(i)}$ & {$[0 / 0]$} & {$[1 / 0]$} & {$[0 / 1]$} & {$[2 / 0]$} & {$[0 / 2]$} & {$[1 / 1]$} & $R$ & $R_{i n v}^{-1}$ & $I\left(\alpha_{1}, \nu, \eta\right)$ \\
\hline$\eta_{c}$ & $-0.7(-0.9)$ & 0.00 & 0.362 & -0.266 & 0.183 & 0.055 & 0.144 & 0.168 & 0.160 & -0.119 \\
$\alpha_{1}$ & $-1.1(-1.7)$ & 0.50 & 0.217 & 0.280 & 0.467 & 0.399 & 0.350 & 0.336 & 0.342 & 0.342 \\
$\alpha_{11}$ & $-0.8(-1.1)$ & 0.00 & -0.362 & -0.266 & 0.109 & -0.021 & -0.157 & -0.180 & -0.170 & -0.140 \\
$\Phi$ & $-0.4(-0.4)$ & 0.50 & 0.421 & 0.427 & 0.642 & 0.657 & 0.479 & 0.474 & 0.474 & 0.518 \\
\hline
\end{tabular}

These are $\Delta_{1}=0.921$ for $n=0$ and $\Delta_{1}=0.997$ for $n=1$. We have calculated yet once the other exponents in the group through the scaling laws using the above values of $\Delta_{1}$ and the standard values of $\nu$ and $\eta$ from reference [6]. These are $\nu=0.588, \eta=0.027$ for $n=0$ and $\nu=0.630, \eta=0.031$ for $n=1$. The resulting values are given in the last row of the tables 1 and 2. One sees the reasonable agreement of the latter with the results obtained from each individual expansion. Deviations of these values from the other resummation estimates could serve as rough indications of the present numerical accuracy.

The situation is more complicated in the second group of exponents, $\alpha_{1}$, $\alpha_{11}$, and $\Phi$, as they exhibit bad convergence properties. Their estimates, along with the renormalization function $\eta_{c}^{s p}$ are given in the tables 3 and 4 . One should be cautious in relying upon the estimates arising from individual series expansions having apparently divergent character, like in the case of the crossover exponent $\Phi$. In this group the exponent $\alpha_{1}$ has the estimates with the least scattering. The best series convergence has $\alpha_{1}^{-1}$, and we accept the corresponding values $\alpha_{1}=0.342$ for $n=0$ and $\alpha_{1}=0.279$ for $n=1$. The values of $\alpha_{11}, \Phi$ and $\eta_{c}^{s p}$ listed in the last rows of tables 3 and 4 have been obtained from the accepted values of $\alpha_{1}, \nu$ and $\eta$.

Our numerical values of the surface critical exponents gathered in tables 1-4 generally are in reasonable agreement both with previous estimates

Table 4. Special transition surface critical exponents associated to the renormalization-group function $\eta_{c} . n=1, u^{*}=$ 1.597 .

\begin{tabular}{|c|l|l|l|l|l|l|l|l|l|c|}
\hline Index & $O_{1(i)} / O_{2(i)}$ & {$[0 / 0]$} & {$[1 / 0]$} & {$[0 / 1]$} & {$[2 / 0]$} & {$[0 / 2]$} & {$[1 / 1]$} & $R$ & $R_{\text {inv }}^{-1}$ & $I\left(\alpha_{1}, \nu, \eta\right)$ \\
\hline$\eta_{c}$ & $-0.7(-1.1)$ & 0.00 & -0.472 & -0.321 & 0.168 & 0.052 & 0.200 & 0.230 & 0.215 & -0.144 \\
$\alpha_{1}$ & $-1.4(-2.9)$ & 0.50 & 0.131 & 0.230 & 0.393 & 0.304 & 0.284 & 0.268 & 0.279 & 0.279 \\
$\alpha_{11}$ & $-0.9(-1.6)$ & 0.00 & -0.472 & -0.321 & 0.042 & 0.153 & -0.226 & -0.253 & 0.237 & -0.182 \\
$\Phi$ & $-0.4(-0.4)$ & 0.50 & 0.397 & 0.407 & 0.649 & 0.661 & 0.470 & 0.463 & 0.463 & 0.539 \\
\hline
\end{tabular}


Table 5. Monte Carlo estimates of special transition exponents.

\begin{tabular}{|c|c|c|}
\hline \multicolumn{3}{|c|}{$n=0$} \\
\hline$\gamma_{11}$ & $0.805(15)$ & Meirovitch, Livne, 1988 [28] \\
& $0.714(6)$ & Hegger, Grassberger, 1994 [32] \\
$\gamma_{1}$ & $1.304(6)$ & Meirovitch, Livne, 1988 [28] \\
& $1.230(2)$ & Hegger, Grassberger, 1994 [32] \\
& $0.530(7)$ & Meirovitch, Livne, 1988 [28] \\
& $0.496(4)$ & Hegger, Grassberger, 1994 [32] \\
\hline \multicolumn{3}{|c|}{$n=1$} \\
\hline$\beta_{1}$ & $0.18(2)$ & Landau, Binder, 1990 [29] \\
& 0.22 & Vendruscolo et al. 1992 [30] \\
& $0.237(5)$ & Ruge et al. 1993 [31] \\
& $0.2375(15)$ & Ruge, Wagner 1995 [44] \\
$\gamma_{11}$ & $0.96(9)$ & Landau, Binder, 1990 [29] \\
& $0.788(1)$ & Ruge, Wagner 1995 [44] \\
$\gamma_{1}$ & $1.41(14)$ & Landau, Binder, 1990 [29] \\
& $1.328(1)$ & Ruge, Wagner 1995 [44] \\
& $0.59(4)$ & Landau, Binder, 1990 [29] \\
& 0.74 & Vendruscolo et al. 1992 [30] \\
& $0.461(15)$ & Ruge et al. 1993 [31] \\
\hline
\end{tabular}

based on the $\epsilon$ expansion as well as with those obtained by other means. For comparisons we refer to reference [15] where the $\epsilon$ expansion estimates and other methods' data up to 1985 are collected, and to the table 5 for more recent results. Note, however, that our estimates for the crossover exponent $\Phi$ are definitively lower than the values $\Phi(n=1) \simeq 0.68$ and $\Phi(n=0) \simeq 0.67$ quoted in reference [15]. These were obtained by setting $\epsilon=1$ in the $\epsilon$ expansion of $\Phi$ to order $\epsilon^{2}$. On the other hand, recent Monte Carlo simulations yielded the significantly lower estimates $\Phi(1)=0.461 \pm 0.015[31]$, $\Phi(0)=0.530 \pm 0.007[28]$, and $\Phi(0)=0.496 \pm 0.005$ [32]. Our results $\Phi(1) \simeq 0.54$ and $\Phi(0) \simeq 0.52$ are fairly close to these values. This indicates that the crossover exponents $\Phi$ for $d=3$ and $n=0,1$ are indeed smaller than previously thought. Let us also note that when the $\epsilon$ expansion to order $\epsilon^{2}$ is extrapolated to $d=3$ by means of more elaborate techniques (e.g, Padé-Borel resummation), estimates for $\Phi(0)$ and $\Phi(1)$ as low as ours may be obtained.

\section{Acknowledgements}

The author is grateful the Alexander von Humboldt-Stiftung for the research fellowship which has made the present work possible. Partial financial support by the Deutsche Forschungsgemeinschaft through SFB 237 is gratefully acknowledged. I thank Prof. Dr. H. W. Diehl and his group for hospitality at Universität-Gesamthochschule Essen where the main part of this work has been performed.

\section{References}

[1] Wilson K.G., Kogut J. The renormalization group and the $\epsilon$ expansion. // Phys. Rep., 1974, vol. 12C, No 1, p. 75-314. 
[2] "Phase transitions and critical phenomena", ed. by C.Domb and M.S.Green, vol. 6. New York, Academic Press, 1976.

[3] Zinn-Justin J. Euclidean field theory and critical phenomena. New York, Oxford University Press, 1989.

[4] Recent historical survey can be found in: Schweber S.S. Physics, community and the crisis in physical theory. // Phys. Today, 1993, vol. 46, No 11 , p. 34-40.

[5] Baker G.A.,Jr., Nickel B.G., Meiron D.I. Critical indices from perturbation analysis of the Callan-Symanzik equation. // Phys. Rev. B, 1978, vol. 17, No 3, p. 1365-1374.

[6] Le Guillou J.C., Zinn-Justin J. Critical exponents from field theory. // Phys. Rev. B, 1980, vol. 21, No 9, p. 3976-3998.

[7] Gorishny S.G., Larin S.A., Tkachov F.V. $\epsilon$ expansion for critical exponents: the $O\left(\epsilon^{5}\right)$ approximation. // Phys. Lett. A, 1984, vol. 101, No 3, p. $120-123$.

[8] Le Guillou J.C., Zinn-Justin J. Accurate critical exponents from the $\epsilon$ expansion. // J. Physique. Lett., 1985, vol. 46, No 4, p. L-137-L-141.

[9] Kleinert H., Neu J., Schulte-Frohlinde V., Chetyrkin K.G., Larin S.A. Five-loop renormalization group functions of $O(n)$-symmetric theory and $\epsilon$ expansions of critical exponents up to $\epsilon^{5}$. // Phys. Lett. B, 1991, vol. 272 , No 1, p. 39-44.

[10] Bagnuls C., Bervillier C. Nonasymptotic critical behavior from field theory at $d=3$ : The disordered-phase case. // Phys. Rev. B, 1985, vol. 32, No 11, p. 7209-7231.

[11] Bagnuls C., Bervillier C., Meiron D.I., Nickel B.G. Nonasymptotic critical behavior from field theory at $d=3$. II. The ordered-phase case. // Phys. Rev. B, 1987, vol. 35, No 7, p. 3585-3607.

[12] Schloms R., Dohm V. Minimal renormalization without $\epsilon$-expansion: critical behavior in three dimensions. // Nucl. Phys. B, 1989, vol. 328, No 3, p. 639-663.

[13] Halfkann F.J., Dohm V. Minimal renormalization without $\epsilon$-expansion. Amplitude functions in three dimensions below $T_{c}$. // Z. Phys. B, 1992, vol. 89 , No 1, p. 79-87.

[14] For a general review of critical behaviour at surfaces see: Binder K. Critical behaviour at surfaces. In: "Phase transitions and Critical Phenomena", ed. by C.Domb and J.L.Lebowitz, vol. 8, p. 1-144. London, Academic Press, 1983; and references [15,16].

[15] Diehl H.W. Field-theoretical approach to critical behavior at surfaces. In: "Phase transitions and Critical Phenomena", ed. by C.Domb and J.L.Lebowitz, vol. 10, p. 75-267. London, Academic Press, 1986. This original article contains an exhaustive list of references up to 1986. For most recent review see reference [16].

[16] Diehl H.W. The theory of boundary critical phenomena. In: Proceedings of the Third Intrnational Conference "Renormalization Group96", Dubna, Russia, 1996. Singapore, World Scientific, 1997. (Preprint cond-mat/9610143).

[17] Diehl H.W., Shpot M. Surface critical behavior in fixed dimensions $d<4$ : Nonanalyticity of critical surface enhancement and massive field theory approach. // Phys. Rev. Lett., 1994, vol. 73, No 25, p. 3431-3434.

[18] Parisi G. Field-theoretic approach to second-order phase transitions in two- and three-dimensional systems. // J. Stat. Phys., 1980, vol. 23, No 1, p. $49-82$.

[19] Parisi G. Statistical field theory. Redwood, Addison-Wesley, 1988. 
[20] Itzykson C., Drouffe J.-M. Statistical field theory. Cambridge, Cambridge University Press, 1989.

[21] Pawley G.S., Swendsen R.H., Wallace D.J., Wilson K.G. Monte Carlo renormalization-group calculations of critical behavior in the simplecubic Ising model. // Phys. Rev. B, 1984, vol. 29, No 7, p. 4030-4040.

[22] Liu A.J., Fisher M.E. The three-dimensional Ising model revisited numerically. // Physica A, 1989, vol. 156, No 1, p. 35-76.

[23] Münster G., Heitger J. Field-theoretic calculation of the universal amplitude ratio of correlation lendths in 3D-Ising systems. // Nucl. Phys. B, 1994, vol. 424, No 3, p. 582-595; Gutsfeld C., Küster J., Münster G. Calculation of universal amplitude ratios in three-loop order. Preprint cond-mat/9606091.

[24] Shpot N.A. Critical behavior of the $m n$-component field model in three dimensions. II. Three-loop results. // Phys. Lett. A, 1989, vol. 142, No 8,9, p. 474-478; Critical behavior of the $m n$-component field model in three dimensions. Phys. Lett. A, 1988, vol. 133, No 3, p. 125-127.

[25] Mayer I.O., Sokolov A.I., Shalayev B.N. Critical exponents for cubic and impure uniaxial crystals: most accurate (?) theoretical values. // Ferroel., 1989, vol. 95, No 1, p. 93-96.

[26] Bervillier C., Shpot M. Universal amplitude combinations of the threedimensional random Ising system. // Phys. Rev. B, 1992, vol. 46, No 2, p. $955-968$.

[27] Holovatch Yu., Shpot M. Critical exponents of random Ising-like systems in general dimensions. // J. Stat. Phys., 1992, vol. 66, No 3/4, p. 867-883; Critical behaviour of dilute Ising-like systems in non-integer dimensions. Preprint ITP-90-48E, Kiev-1990.

[28] Meirovitch H., Livne S.J. Computer simulation of long polymers adsorbed on a surface. II. Critical behavior of a single self-avoidoing walk. // Chem. Phys., 1988, vol. 88, No 7, p. 4507-4515.

[29] Landau D.P., Binder K. Monte Carlo study of surfce phase transitions in the three-dimensional Ising model. // Phys. Rev. B, 1990, vol. 41, No 7, p. 4633-4645.

[30] Vendruscolo M., Rovere M., Fasolino A. Magmetic-phase transitions of Ising surfaces with modified surface-bulk coupling: a Monte Carlo study. // Europhys. Lett., 1992, vol. 20, No 6, p. 547-552.

[31] Ruge C., Dunkelmann S., Wagner F., Wulf J. Study of the threedimensional Ising model on film geometry with the cluster Monte Carlo method. // J. Stat. Phys., 1993, vol. 73, No 1/2, p. 293-317.

[32] Hegger R., Grassberger P. Chain polymers near an adsorbing surface. // J. Phys. A, 1994, vol. 27, No 12, p. 4069-4081.

[33] Diehl H.W., Shpot M., to be published.

[34] For background and references see E. Eisenriegler, Polymers near surfaces. Singapore, World Scientific, 1993.

[35] Brézin E., Le Guillou J.C., Zinn-Justin J. Field theoretical approach to critical phenomena. In reference [2], p. 125-247.

[36] Amit D. Field theory, the renormalization group, and critical phenomena. New York, Mc Graw-Hill, 1978.

[37] Diehl H.W., Dietrich S. Field-theoretical approach to multicritical behavior near free surfaces. // Phys. Rev. B, 1981, vol. 24, No 5, p. 2878-2880; Multicritical behaviour at surfaces. Z. Phys. B, 1983, vol. 50, No 2, p. 117-129.

[38] Prudnikov A., Brychkov Yu.A., Marichev O.I. Integrals and Series. Vol. 1: Elementary Functions. New York, Gordon and Breach, 1986. 
[39] Handbook of Mathematical Functions. Ed. by M.Abramowitz, I.Stegun. New York, Dover, 1965.

[40] Baker G.A.,Jr., Essentials of Padé approximants. New York, Academic Press, 1975.

[41] Baker G.A.,Jr., Nickel B.G., Green M.S., Meiron D.I. Ising-model critical indices in three dimensions from the Callan-Symanzik equation. // Phys. Rev. Lett., 1976, vol. 36, No 23, p. 1351-1354.

[42] Jug G. Critical behavior of disordered spin systems in two and three dimensions. // Phys. Rev. B, 1983, vol. 27, No 1, p. 609-612.

[43] Shpot N.A. Critical behaviour of the anisotropic mn-component field model in three dimensions. Preprint ITF-88-16R, Kiev-1988 (in Russian).

[44] Ruge C., Wagner F. Critical parameters for the $d=3$ Ising model in a film geometry. // Phys. Rev. B, 1995 , vol. 52, No 6, p. 4209-4216.

\title{
СПЕЦІАЛЬНИЙ ПОВЕРХНЕВИЙ ПЕРЕХІД: МАСИВНА ТЕОРІЯ ПОЛЯ І КРИТИЧНІ ПОКАЗНИКИ У ТРЬОХ BИMIPAX
}

\begin{abstract}
М.Шпот
Поверхнева критична поведінка досліджується безпосередньо у фіксованих розмірностях простору $d=4-\epsilon<4$ без звертання до $\epsilon$-розкладу. Метод масивної теорії поля узагальнений відповідно до опису стандартної напівобмеженої $n$-векторної моделі з поверхневим членом $\frac{1}{2} c_{0} \int_{\partial V} \phi^{2}$. Вводиться адитивний зсув параметра $c_{0}$, що описує специфіку поверхневих взаємодій, аналогічний до зсуву "маси" в суперперенормовних теоріях поля. Явні двопетлеві розрахунки у тривимірному просторі дають чисельні оцінки поверхневих критичних показників спеціального фазового переходу. Отримані результати добре узгоджуються з даними найсучасніших комп'ютерних розрахунків типу Монте-Карло.
\end{abstract}

